\title{
Telescope Array 10-Year Monocular Spectrum Measurement
}

\section{Douglas R. Bergman ${ }^{a, *}$ and Greg Furlich ${ }^{a, *}$ on behalf of the Telescope Array Collaboration}

(a complete list of authors can be found at the end of the proceedings)

${ }^{a}$ Dept. of Physics \& Astronomy and High Energy Astrophysics Inst., University of Utah, Utah, USA

E-mail: bergman@physics.utah.edu

Telescope Array (TA) is the largest cosmic ray detector in the northern hemisphere. We present a measurement of the cosmic ray energy spectrum for energies above $10^{17.5} \mathrm{eV}$ using fluorescence telescopes in monocular mode. A novel weather classification scheme using machine learning was used to select data parts with good weather to ensure the quality of the fluorescence data. The data from the Black Rock Mesa (BR) and Long Ridge (LR) fluorescence telescope stations were analysed separately in monocular mode, with the calculated fluxes combined into a single spectrum. The 10-year monocular combined cosmic ray energy spectrum is in excellent agreement with previous measurements from the northern hemisphere. We present fits of the combined spectrum to a series of broken power law models. A thrice-broken power law is observed to be the best fit considering the Poisson deviance per degrees of freedom. The three breaks suggest an additional feature of the spectrum between the previously observed Ankle at $10^{18.7} \mathrm{eV}$ and the GZK suppression at $10^{19.8} \mathrm{eV}$.

$37^{\text {th }}$ International Cosmic Ray Conference (ICRC 2021)

July 12th - 23rd, 2021

Online - Berlin, Germany

\footnotetext{
*Presenter
} 


\section{Introduction}

The Telescope Array (TA) Cosmic Ray Observatory is the largest cosmic ray detector operating in the northern hemisphere[1]. To better understand the nature of cosmic ray populations at the highest energies and the mechanisms that influence their propagation through the universe, we continue to measure the cosmic ray energy spectrum.

TA has passed 11 years of operation. As a followup to the previous TA FD monocular measurements with 3.5 years[2] and 7.5 years[3] of data and the latest measurement made by the TA SDs[4], we preform a spectrum calculation using nearly 10 years of data from the BR and LR FD stations in monocular mode. While the FDs operate with $11 \%$ duty cycle compared to the nearly $100 \%$ duty cycle of the SDs, the FDs observe the longitudinal development in the Extensive Air Showers (EAS) and have less hadronic-model dependency for event reconstruction.

\section{Monocular Reconstruction}

The analysis of events begins by calculating the shower-detector plane (SDP). The normal to the shower detector plane is determined by minimizing

$$
\chi^{2}=\sum_{i=1}^{N_{\text {good }}}\left(\hat{n} \cdot \hat{v}_{i}\right)^{2} N_{\mathrm{pe}, i}
$$

where $\hat{n}$ is the normal to the SDP, $\hat{v}_{i}$ the pointing direction of a good PMT, and $N_{\text {p.e. }, i}$ is the number of photoelectrons is a good PMT.

Next, the geometry of the shower within the SDP is reconstructed by fitting the time vs. angle of observed signals. The fitted form is

$$
t_{i}=t_{0}+\frac{R_{P}}{c} \tan \left(\frac{\pi-\psi-\chi_{i}}{2}\right)
$$

where $t_{i}$ the time of a good tube, $t_{0}$ time when the first light can reach to the detector, $R_{P}$ is the impact parameter, $\psi$ is the angle of the shower with respect to the ground within the SDP, and $\chi_{i}$ is the angle viewed by a good tube from the horizon within the SDP. In this fit, $R_{P}, \psi$, and $t_{0}$ are the floating parameters and there is typically considerable correlation between them, requiring tracks with considerable angular extent to allow a good fit.

Once the geometry is determined, the shower profile is measured using the flux of photons observed by the detector, the atmospheric attenuation given the atmospheric properties, and the average fluorescence yield. The shower profile is fit to a Gaisser-Hillas[5] profile as a function of shower slant depth $X$ :

$$
N_{\text {ch }}=N_{\max }\left(\frac{X-X_{\max }}{X_{0}-X_{\max }}\right)^{\frac{X-X_{\max }}{\Lambda}} \exp \left(\frac{X_{\max }-X}{\Lambda}\right)
$$

where $N_{\max }$ is the size of the shower at maximum, $X_{\max }$ is the slant depth of the maximum of the shower (measured in $\mathrm{g} / \mathrm{cm}^{2}$ ),$X_{0}$ is an unobservable "beginning" of the shower and $\Lambda$ is the decay parameter of the shower. The calorimetric energy of the shower is determined by integrating the energy deposited by the shower particles over the full extent of the shower

$$
E_{\mathrm{cal}}=\left.\int_{X_{0}}^{\infty} \frac{d E_{\mathrm{dep}}}{d X}\right|_{X} d X
$$


The missing energy, energy carried off by neutrinos and muons which do not deposit most of their energy in the atmosphere is added back in by a correction calculated from CORSIKA[6]

$$
\frac{E_{\mathrm{cal}}}{E_{0}}=-0.5717+0.1416 \log _{10}\left(E_{\mathrm{cal}} / \mathrm{eV}\right)-0.003328\left[\log _{10}\left(E_{\mathrm{cal}} / \mathrm{eV}\right)\right]^{2}
$$

We use a new variable $R_{X_{\max }}$ which measures the distance to the brightest part of the shower, as shown to the right. This variable is more relevant for defining the aperture than the impact parameter $X_{\max }$ which may refer to a part of the shower far away from where light is being emitted. See Figure 1.

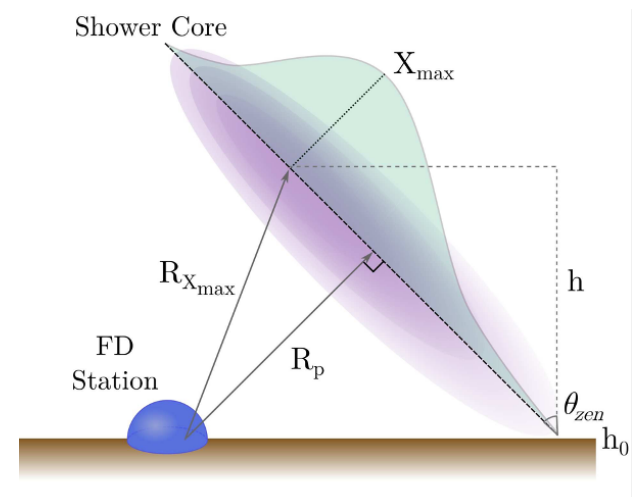

Figure 1: Definition of $R_{X_{\max }}$ and its relation to the impact parameter $X_{\max }$.

\section{Event Selection}

From triggered events, a selection was made by various cuts. Cuts at the geometry reconstruction stage are given in Table 1. Cuts at the profile reconstruction phase are given in Table 2.

Good weather run segements were chose according to at novel neutral-network weather classification scheme. Using the fact that BRM and LR PMTs are DC connected and thus can provide a measurement of the background light level, one can make a movie of the night sky with one-minute long frames, and look for the passage of stars and/or clouds across the field-of-view. A neutralnetwork was designed and trained to recognize data parts which were either clear or cloudy. The data presented here comes only from the clear weather set. The good weather run time, including deadtime estimates is given in Table 3. Note that time when both detectors were operating and able to view events in tandem is also given.

With these cuts and taking data from good weather days results in the event energy distribution shown in Figure 2.

\section{Aperture and Exposure Calculation}

The instantaneous aperture of the detector of the detector was determined by Monte Carlo simulation. The showers thrown in the simulation were drawn from a shower library of CORSIKAsimulated proton and iron showers. The ratio of proton and iron showers at a particular energy were chosen according to the HiRes/MIA composition fit[7]. The energy was chosen according to the TA SD spectrum presented at the 2015 ICRC [8]. The detector response was simulated according 
Event Geometry Reconstruction Cuts

\begin{tabular}{lr}
\hline \hline Good PMT Fraction & $N_{\text {good PMT }} / N_{\text {PMT }} \geq 3.5 \%$ \\
Number of good PMTs & $N_{\text {good PMT }} \geq 6$ \\
NPE per Degree & $N_{\text {pe }} / \Delta \theta>25 \mathrm{deg}^{-1}$ \\
Pseudo-distance (inverse angular speed) & $>1.5 \mathrm{~km}$ \\
SDP Angle (w.r.t. vertical) & $\zeta<80^{\circ}$ \\
Impact Parameter & $R_{P} \geq 0.5 \mathrm{~km}$ \\
Shower angle in SDP & $\psi<130^{\circ}$ \\
Shower angle uncertainty & $\sigma_{\psi}<36^{\circ}$ \\
Timing Fit & Successful \\
& $\chi^{2} /$ ndf $<10$ \\
Track Length & $\Delta \theta>10^{\circ}$ \\
& $\Delta \theta 7^{\circ}$ (only in lower mirror) \\
Zenith Angle & $\theta_{\text {zen }}<70^{\circ}$ \\
Trigger Time w/i Frame & $t_{0}<25.6 \mu \mathrm{s}$ \\
Crossing Time & $\Delta t<6 \mu$ s for $R_{P}<5 \mathrm{~km}$
\end{tabular}

Table 1: Event selection cuts applied at the geometry reconstruction phase.

Profile Reconstruction Fits

\begin{tabular}{lr}
\hline \hline Profile Fit & Successful \\
First Slant Depth Observed & $150 \mathrm{~g} / \mathrm{cm}^{2} \leq X_{1} \leq 1200 \mathrm{~g} / \mathrm{cm}^{2}$ \\
Slant Depth Extent Observed & $\Delta X \geq 150 \mathrm{~g} / \mathrm{cm}^{2}$ \\
Bracketing & $X_{1} \leq X_{\max } \leq X_{\text {last }}$
\end{tabular}

Table 2: Event selection cuts applied at the profile reconstruction phase.

\begin{tabular}{lrrrrrr} 
& $\begin{array}{r}\text { Operating } \\
\text { Nights }\end{array}$ & $\begin{array}{r}\text { Duty } \\
\text { Cycle }\end{array}$ & $\begin{array}{r}\text { Ontime } \\
{[\mathrm{h}]}\end{array}$ & $\begin{array}{r}\text { Deadtime } \\
{[\mathrm{h}]}\end{array}$ & $\begin{array}{r}\text { Deadtime } \\
\text { Fraction }\end{array}$ & $\begin{array}{r}\text { Livetime } \\
{[\mathrm{h}]}\end{array}$ \\
\hline \hline BRM & 1593 & $10.97 \%$ & 9288.19 & 574.16 & $6.18 \%$ & 8714.03 \\
LR & 1493 & $9.03 \%$ & 7647.41 & 555.79 & $7.26 \%$ & 7091.63 \\
BRM $\cap$ LR & 1378 & & 6876.40 & & $13.00 \%$ & 5982.47
\end{tabular}

Table 3: Runtime statistics for the two detectors and their overlap.

to daily calibration measurements and an average atmospheric model. The total exposures were fit to the form

$$
\xi=p_{1}\left(1-\exp \left[-\frac{\log _{10}\left(E / \mathrm{eV}-p_{2}\right.}{p_{3}}\right]\right)
$$

for most of the aperture to smooth out the effect of MC statistics at the highest energies. The resulting exposures are shown in Figure 3

To verify the accuracy of the exposure calculation we present three comparisons of simulated data distributions to the same distribution in the actual data. We chose three that are most relevant to the exposure: $R_{X_{\max }}, \psi$, and the SDP angle. These comparisons are shown in Figure 4 


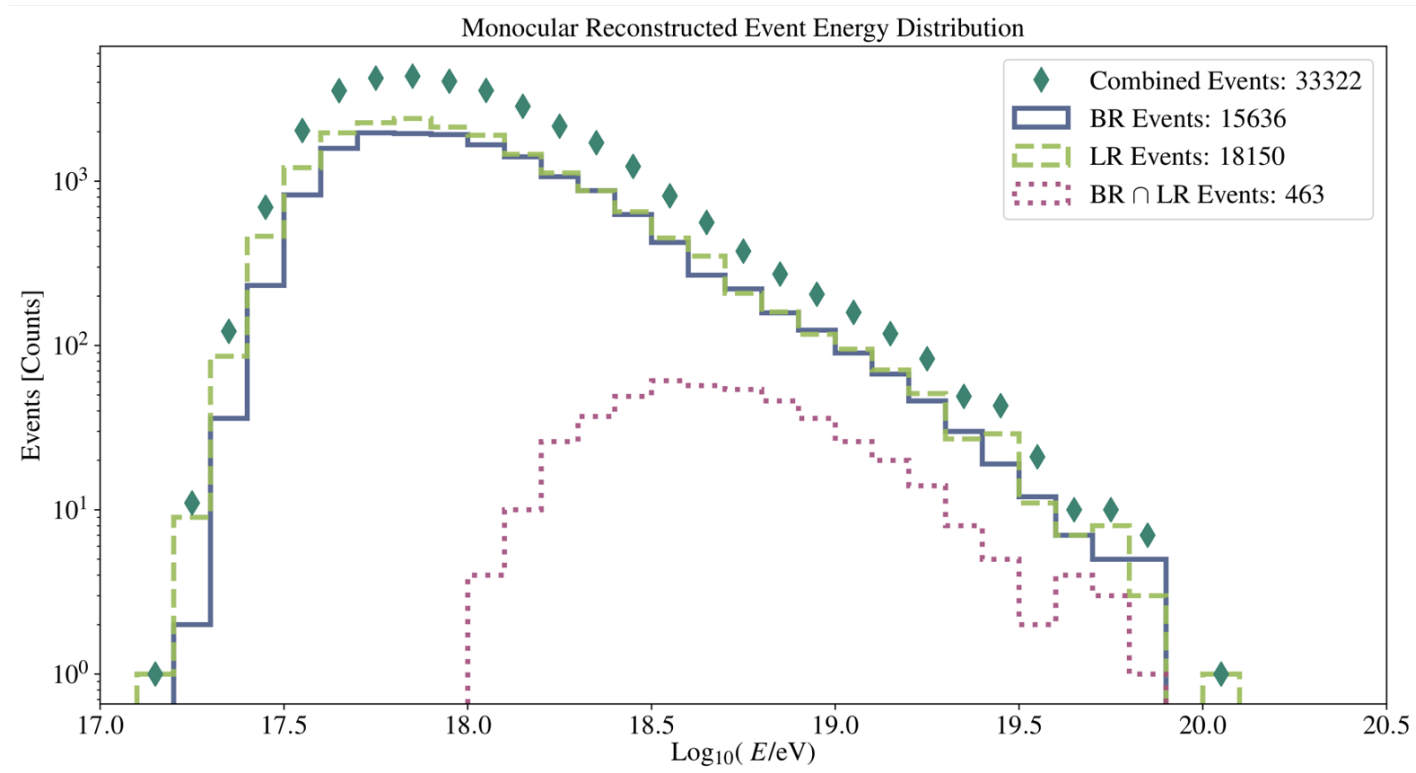

Figure 2: The energy distribution of events included in this analysis. BR and LR events are seen by each sight singly. BRM $\cap$ LR events are seen by both sights and plotted as the geometric mean of the two measured energies.

\section{Spectrum Calculation}

Using the data and exposure presented, we calculate the following spectrum of UHECRs. We present it in Figure 5 along with the TA SD spectrum from the 2019 ICRC[4] for comparison.

We have fit the spectrum to a series of once, twice and thrice-broken power law models. The results of the fits are shown in Figure 6. For the two-break point fits, two separate solutions were found with very nearly the same chi-squared value, so we present both. The quality of the three break point fit indicates the presence of a feature between the Ankle and the GZK suppression, which has been observed in other analyses.

\begin{tabular}{l|rrrr} 
& Single & Double A & Double B & Triple \\
\hline \hline$J_{18} / 10^{-30}$ & $2.22 \pm 0.01$ & $2.22 \pm 0.01$ & $2.22 \pm 0.01$ & $2.22 \pm 0.01$ \\
$\left(\mathrm{eV} \mathrm{m} \mathrm{sr} \mathrm{s}^{-1}\right.$ & & & & \\
$\gamma_{1}$ & $-3.29 \pm 0.01$ & $-3.29 \pm 0.01$ & $-3.29 \pm 0.01$ & $-3.29 \pm 0.01$ \\
$\log _{10}\left(E_{1} / \mathrm{eV}\right)$ & $18.68 \pm 0.04$ & $18.74 \pm 0.03$ & $18.72 \pm 0.05$ & $18.78 \pm 0.04$ \\
$\gamma_{2}$ & $-2.79 \pm 0.05$ & $-2.62 \pm 0.06$ & $-2.70 \pm 0.05$ & $-2.49 \pm 0.15$ \\
$\log _{10}\left(E_{2} / \mathrm{eV}\right)$ & & $19.46 \pm 0.10$ & $19.83 \pm 0.04$ & $19.20 \pm 0.11$ \\
$\gamma_{3}$ & & $-3.8 \pm 0.4$ & $-8.0 \pm 2.7$ & $-3.04 \pm 0.19$ \\
$\log _{10}\left(E_{3} / \mathrm{eV}\right)$ & & & & $19.85 \pm 0.01$ \\
$\gamma_{3}$ & & & & $-7.7 \pm 2.4$ \\
$D / \mathrm{ndf}$ & $43.5 / 26$ & $22.69 / 24$ & $23.29 / 24$ & $17.74 / 22$
\end{tabular}

Table 4: Parameters resulting from broken-powerlaw fits to the monocular spectrum. 


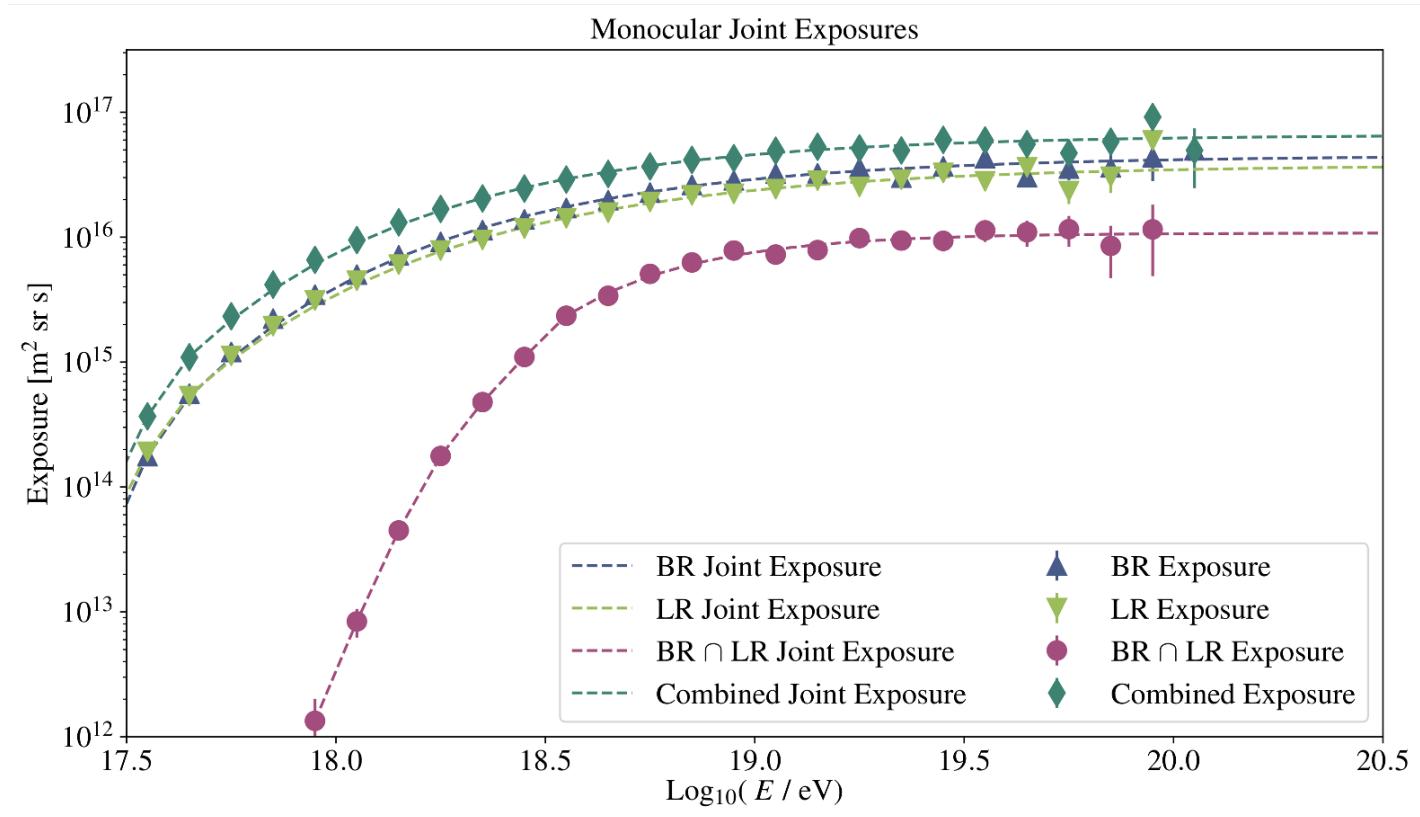

Figure 3: The exposure of the three components of the detector: BRM and LR solely and BRM\&LR together. The sum of the three exposures is also shown.

\section{Acknowledgments}

The Telescope Array experiment is supported by the Japan Society for the Promotion of Science(JSPS) through Grants-in-Aid for Priority Area 431, for Specially Promoted Research JP21000002, for Scientific Research (S) JP19104006, for Specially Promoted Research JP15H05693, for Scientific Research (S) JP15H05741 and JP19H05607, for Science Research (A) JP18H03705, for Young Scientists (A) JPH26707011, and for Fostering Joint International Research (B) JP19KK0074, by the joint research program of the Institute for Cosmic Ray Research (ICRR), The University of Tokyo; by the Pioneering Program of RIKEN for the Evolution of Matter in the Universe (r-EMU); by the U.S. National Science Foundation awards PHY-1404495, PHY-1404502, PHY-1607727, PHY-1712517, PHY-1806797 and PHY-2012934; by the National Research Foundation of Korea (2017K1A4A3015188, 2020R1A2C1008230, \& 2020R1A2C2102800) ; by the Ministry of Science and Higher Education of the Russian Federation under the contract 075-15-2020778, RFBR grant 20-02-00625a (INR), IISN project No. 4.4501.18, and Belgian Science Policy under IUAP VII/37 (ULB). This work was partially supported by the grants ofThe joint research program of the Institute for Space-Earth Environmental Research, Nagoya University and Inter-University Research Program of the Institute for Cosmic Ray Research of University of Tokyo. The foundations of Dr. Ezekiel R. and Edna Wattis Dumke, Willard L. Eccles, and George S. and Dolores Doré Eccles all helped with generous donations. The State of Utah supported the project through its Economic Development Board, and the University of Utah through the Office of the Vice President for Research. The experimental site became available through the cooperation of the Utah School and Institutional Trust Lands Administration (SITLA), U.S. Bureau of Land Management (BLM), and the U.S. Air Force. We appreciate the assistance of the State of Utah and Fillmore offices of the BLM in crafting the Plan of Development for the site. Patrick A. Shea assisted the collaboration with valuable advice and supported the collaborationâ $€^{\mathrm{TM}_{S}}$ efforts. The people and the officials of Millard County, Utah have been a source of steadfast and warm support for our work which we greatly 

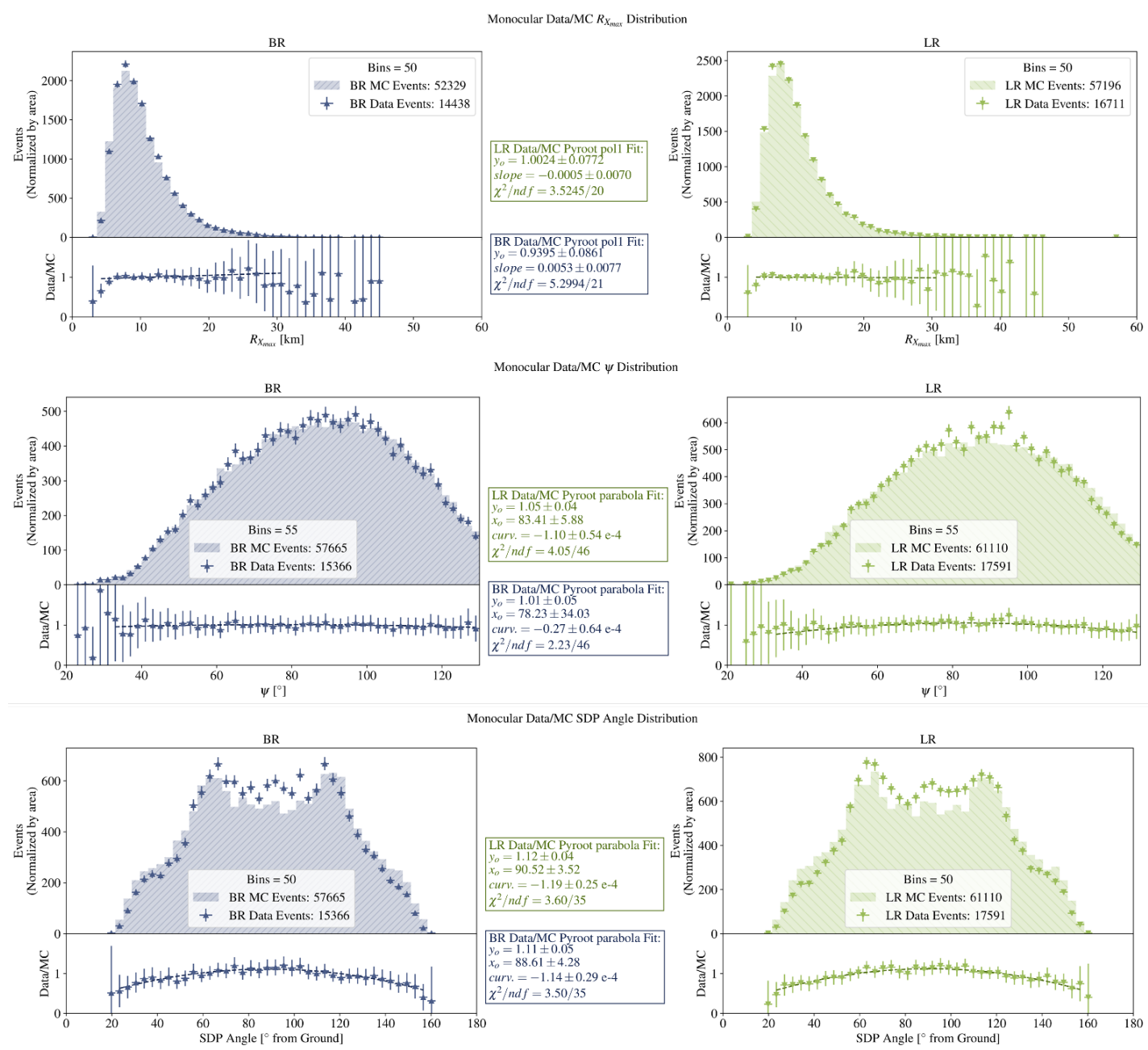

Figure 4: Data/MC Comparisons to verify the quality of the exposure calculation. Shown are comparisons of $R_{X_{\max }}, \psi$, and the SDP angle distributions.

appreciate. We are indebted to the Millard County Road Department for their efforts to maintain and clear the roads which get us to our sites. We gratefully acknowledge the contribution from the technical staffs of our home institutions. An allocation of computer time from the Center for High Performance Computing at the University of Utah is gratefully acknowledged.

\section{References}

[1] H. Kuwai et al., Nucl. Phys 175-176 (2008) 221.

[2] T. Abu-Zayyad et al., Astropart. Phys. 48 (2013) 16.

[3] R. Abbasi et al., Astropart. Phys. 80 (2016) 131.

[4] D. Ivanov, PoS(ICRC2019)298.

[5] T.K Gaiser, A.M. Hillas, Proc. of the 15th ICRC (Plovdiv), v. 8, p. 343. 


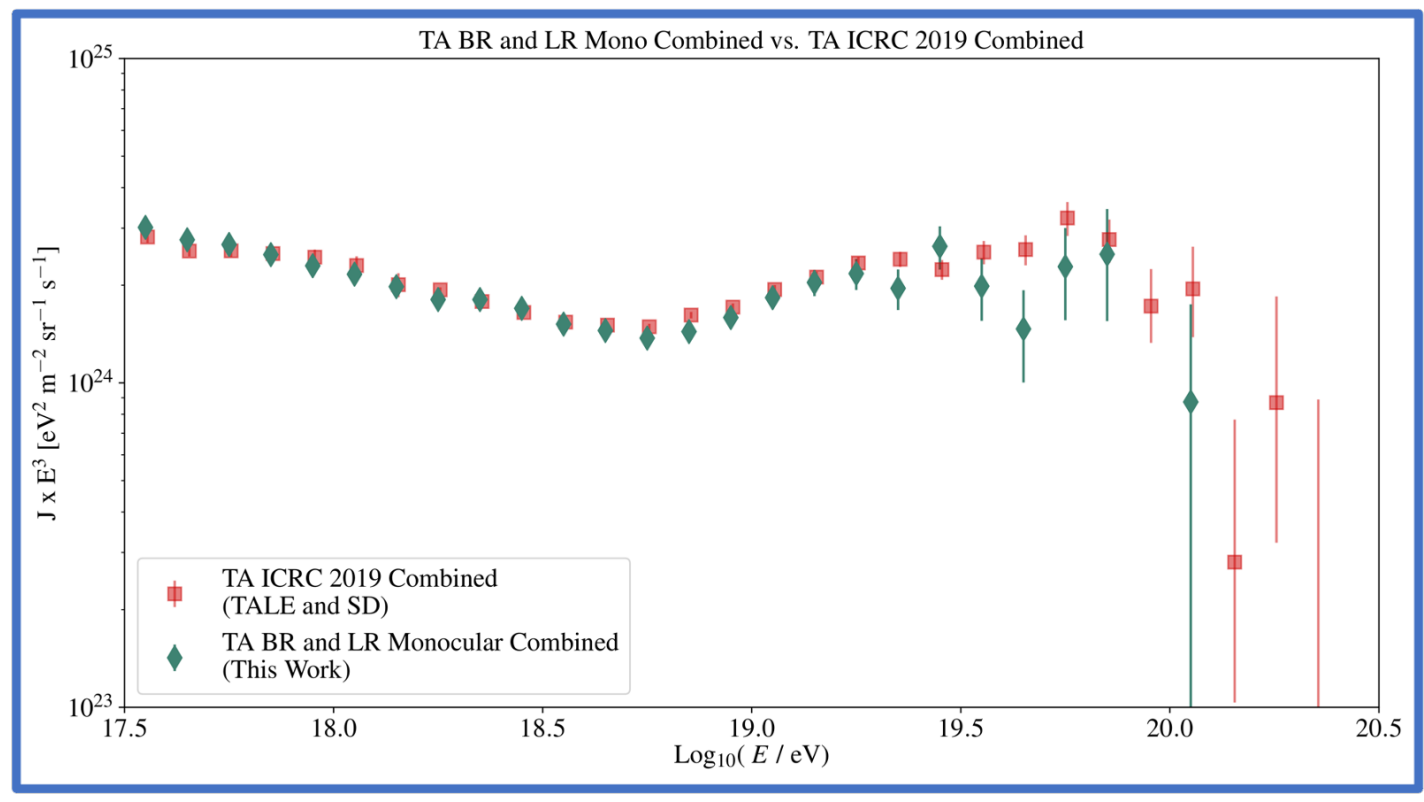

Figure 5: The spectrum of cosmic rays measured in this result. Shown for comparison with the TA SD spectrum presented at the 2019 ICRC.

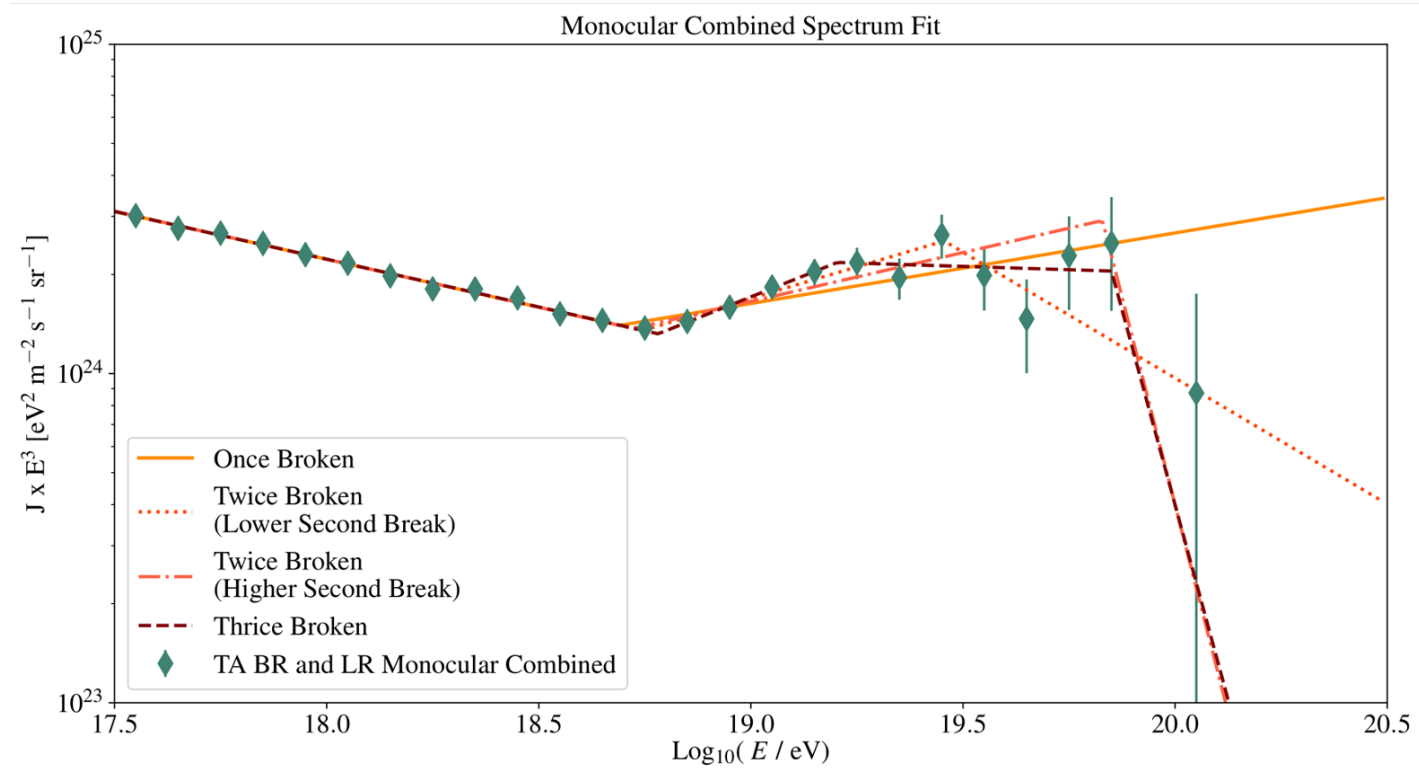

Figure 6: Four power-law fits to the measured spectrum. The parameters of the fits are given in Table 4.

[6] D. Heck et al., CORSIKA: A Monte Carlo Code to Simulate Extensive Air Showers, FZKA 6019.

[7] T. AbuZayyad et al., Phys. Rev. Lett. 84 (2000) 4276.

[8] D. Ivanov, PoS(ICRC2015)349. 


\section{Full Authors List: Telescope Array Collaboration}

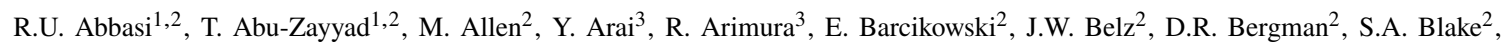
I. Buckland ${ }^{2}$, R. $\mathrm{Cady}^{2}$, B.G. Cheon ${ }^{4}$, J. Chiba ${ }^{5}$, M. Chikawa ${ }^{6}$, T. Fujii ${ }^{7}$, K. Fujisue ${ }^{6}$, K. Fujita ${ }^{3}$, R. Fujiwara ${ }^{3}$, M. Fukushima ${ }^{6}$, R. Fukushima ${ }^{3}$, G. Furlich ${ }^{2}$, R. Gonzalez ${ }^{2}$, W. Hanlon ${ }^{2}$, M. Hayashi ${ }^{8}$, N. Hayashida ${ }^{9}$, K. Hibino ${ }^{9}$, R. Higuchi ${ }^{6}$, K. Honda ${ }^{10}$, D. Ikeda ${ }^{9}$, T. Inadomi ${ }^{11}$, N. Inoue ${ }^{12}$, T. Ishii ${ }^{10}$, H. Ito ${ }^{13}$, D. Ivanov ${ }^{2}$, H. Iwakura ${ }^{11}$, A. Iwasaki ${ }^{3}$, H.M. Jeong ${ }^{14}$, S. Jeong ${ }^{14}$, C.C.H. Jui ${ }^{2}$, K. Kadota ${ }^{15}$, F. Kakimoto ${ }^{9}$, O. Kalashev ${ }^{16}$, K. Kasahara ${ }^{17}$, S. Kasami ${ }^{18}$, H. Kawai ${ }^{19}$, S. Kawakami ${ }^{3}$, S. Kawana ${ }^{12}$, K. Kawata ${ }^{6}$, I. Kharuk ${ }^{16}$, E. Kido ${ }^{13}$, H.B. Kim ${ }^{4}$, J.H. Kim ${ }^{2}$, J.H. Kim ${ }^{2}$, M.H. Kim ${ }^{14}$, S.W. Kim ${ }^{14}$, Y. Kimura ${ }^{3}$, S. Kishigami ${ }^{3}$, Y. Kubota ${ }^{11}$, S. Kurisu ${ }^{11}$, V. Kuzmin ${ }^{16}$, M. Kuznetsov ${ }^{16,20}$, Y.J. Kwon ${ }^{21}$, K.H. Lee ${ }^{14}$, B. Lubsandorzhiev ${ }^{16}$, J.P. Lundquist ${ }^{2,22}$, K. Machida ${ }^{10}$, H. Matsumiya ${ }^{3}$, T. Matsuyama ${ }^{3}$, J.N. Matthews ${ }^{2}$, R. Mayta ${ }^{3}$, M. Minamino ${ }^{3}$, K. Mukai ${ }^{10}$, I. Myers ${ }^{2}$, S. Nagataki ${ }^{13}$, K. Nakai ${ }^{3}$, R. Nakamura ${ }^{11}$, T. Nakamura ${ }^{23}$, T. Nakamura ${ }^{11}$, Y. Nakamura ${ }^{11}$, A. Nakazawa ${ }^{11}$, E. Nishio ${ }^{18}$, T. Nonaka ${ }^{6}$, H. Oda ${ }^{3}$, S. Ogio $^{3,24}$, M. Ohnishi ${ }^{6}$, H. Ohoka ${ }^{6}$, Y. Oku ${ }^{18}$, T. Okuda ${ }^{25}$, Y. Omura ${ }^{3}$, M. Ono ${ }^{13}$, R. Onogi ${ }^{3}$, A. Oshima ${ }^{3}$, S. Ozawa ${ }^{26}$, I.H. Park ${ }^{14}$, M. Potts ${ }^{2}$, M.S. Pshirkov ${ }^{16,27}$, J. Remington ${ }^{2}$, D.C. Rodriguez ${ }^{2}$, G.I. Rubtsov ${ }^{16}$, D. Ryu ${ }^{28}$, H. Sagawa ${ }^{6}$, R. Sahara ${ }^{3}$, Y. Saito ${ }^{11}$, N. Sakaki ${ }^{6}$, T. Sako ${ }^{6}$, N. Sakurai ${ }^{3}$, K. Sano ${ }^{11}$, K. Sato ${ }^{3}$, T. Seki ${ }^{11}$, K. Sekino ${ }^{6}$, P.D. Shah ${ }^{2}$, Y. Shibasaki ${ }^{11}$, F. Shibata ${ }^{10}$, N. Shibata ${ }^{18}$, T. Shibata ${ }^{6}$, H. Shimodaira ${ }^{6}$, B.K. Shin ${ }^{28}$, H.S. Shin ${ }^{6}$, D. Shinto ${ }^{18}$, J.D. Smith ${ }^{2}$, P. Sokolsky ${ }^{2}$, N. Sone ${ }^{11}$, B.T. Stokes ${ }^{2}$, T.A. Stroman ${ }^{2}$, Y. Takagi ${ }^{3}$,

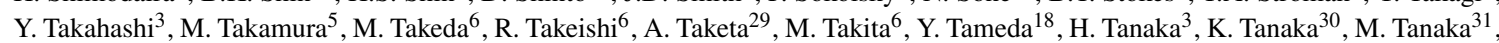
Y. Tanoue ${ }^{3}$, S.B. Thomas ${ }^{2}$, G.B. Thomson ${ }^{2}$, P. Tinyakov ${ }^{16,20}$, I. Tkachev ${ }^{16}$, H. Tokuno ${ }^{32}$, T. Tomida ${ }^{11}$, S. Troitsky ${ }^{16}$, R. Tsuda ${ }^{3}$, Y. Tsunesada ${ }^{3,24}$, Y. Uchihori ${ }^{33}$, S. Udo ${ }^{9}$, T. Uehama ${ }^{11}$, F. Urban ${ }^{34}$, T. Wong ${ }^{2}$, K. Yada $^{6}$, M. Yamamoto ${ }^{11}$, K. Yamazaki ${ }^{9}$, J. Yang ${ }^{35}$, K. Yashiro ${ }^{5}$, F. Yoshida ${ }^{18}$, Y. Yoshioka ${ }^{11}$, Y. Zhezher ${ }^{6,16}$, and Z. Zundel ${ }^{2}$

${ }^{1}$ Department of Physics, Loyola University Chicago, Chicago, Illinois, USA

${ }^{2}$ High Energy Astrophysics Institute and Department of Physics and Astronomy, University of Utah, Salt Lake City, Utah, USA

${ }^{3}$ Graduate School of Science, Osaka City University, Osaka, Osaka, Japan

${ }^{4}$ Department of Physics and The Research Institute of Natural Science, Hanyang University, Seongdong-gu, Seoul, Korea

${ }^{5}$ Department of Physics, Tokyo University of Science, Noda, Chiba, Japan

${ }^{6}$ Institute for Cosmic Ray Research, University of Tokyo, Kashiwa, Chiba, Japan

7 The Hakubi Center for Advanced Research and Graduate School of Science, Kyoto University, KitashirakawaOiwakecho, Sakyo-ku, Kyoto, Japan

${ }^{8}$ Information Engineering Graduate School of Science and Technology, Shinshu University, Nagano, Nagano, Japan

${ }^{9}$ Faculty of Engineering, Kanagawa University, Yokohama, Kanagawa, Japan

${ }^{10}$ Interdisciplinary Graduate School of Medicine and Engineering, University of Yamanashi, Kofu, Yamanashi, Japan

${ }^{11}$ Academic Assembly School of Science and Technology Institute of Engineering, Shinshu University, Nagano, Nagano, Japan

${ }^{12}$ The Graduate School of Science and Engineering, Saitama University, Saitama, Saitama, Japan

13 Astrophysical Big Bang Laboratory, RIKEN, Wako, Saitama, Japan

${ }^{14}$ Department of Physics, SungKyunKwan University, Jang-an-gu, Suwon, Korea

${ }^{15}$ Department of Physics, Tokyo City University, Setagaya-ku, Tokyo, Japan

${ }^{16}$ Institute for Nuclear Research of the Russian Academy of Sciences, Moscow, Russia

${ }^{17}$ Faculty of Systems Engineering and Science, Shibaura Institute of Technology, Minato-ku, Tokyo, Japan

${ }^{18}$ Department of Engineering Science, Faculty of Engineering, Osaka Electro-Communication University, Neyagawashi, Osaka, Japan

${ }^{19}$ Department of Physics, Chiba University, Chiba, Chiba, Japan

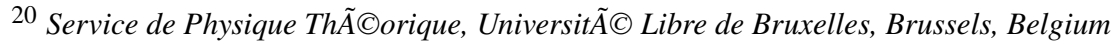

${ }^{21}$ Department of Physics, Yonsei University, Seodaemun-gu, Seoul, Korea

22 Center for Astrophysics and Cosmology, University of Nova Gorica, Nova Gorica, Slovenia

${ }^{23}$ Faculty of Science, Kochi University, Kochi, Kochi, Japan

${ }^{24}$ Nambu Yoichiro Institute of Theoretical and Experimental Physics, Osaka City University, Osaka, Osaka, Japan

${ }^{25}$ Department of Physical Sciences, Ritsumeikan University, Kusatsu, Shiga, Japan

${ }^{26}$ Quantum ICT Advanced Development Center, National Institute for Information and Communications Technology, Koganei, Tokyo, Japan

27 Sternberg Astronomical Institute, Moscow M.V. Lomonosov State University, Moscow, Russia

${ }^{28}$ Department of Physics, School of Natural Sciences, Ulsan National Institute of Science and Technology, UNIST-gil, 
Ulsan, Korea

${ }^{29}$ Earthquake Research Institute, University of Tokyo, Bunkyo-ku, Tokyo, Japan

${ }^{30}$ Graduate School of Information Sciences, Hiroshima City University, Hiroshima, Hiroshima, Japan

${ }^{31}$ Institute of Particle and Nuclear Studies, KEK, Tsukuba, Ibaraki, Japan

${ }^{32}$ Graduate School of Science and Engineering, Tokyo Institute of Technology, Meguro, Tokyo, Japan

33 Department of Research Planning and Promotion, Quantum Medical Science Directorate, National Institutes for Quantum and Radiological Science and Technology, Chiba, Chiba, Japan

${ }^{34}$ CEICO, Institute of Physics, Czech Academy of Sciences, Prague, Czech Republic

${ }^{35}$ Department of Physics and Institute for the Early Universe, Ewha Womans University, Seodaaemun-gu, Seoul, Korea

${ }^{*}$ Deceased 